\title{
Zneite Abtheilung.
}

\section{Vereins - Zeitung, redigirt vom Directorio des Vereins.}

\section{1) Gesetzliche Bestimmungen.}

Aus Veranlassung eines Antrages der königl. Regierung zu Erfurt ist über die Zulässigkeit des Gesuches mehrerer Materialisten, ilnnen den Verkauf des gepülverten Semen Foeni graeci frei zu geben, die gutachtliche Aeusserung der zur Bearbeitung einer neuen Landespharmakopöe niedergesetzten Commission eingeholt worden.

Da danach das genannte Mittel nur in der Vetcrinairpraxis Anwendung findet, ungepülvert aus den Apotheken niemals verlangt, auch nur selten in den Apotheken gestossen, sondern auf Hïhlen in grossen Quantitäten gemahlen, und der Anbau der Pflanzen in einigen Gegenden zum Handel hetrieben wird, so haben wir beschlossen, den Verkauf des gepülverten Foenum graecum ganz frei zu geben und das Mittel in dem, der Verordnung wegen des Debits der Arzneiwaaren vom 16. September 1836 beigefügten Verzeichnisse B. za lüschen.

Die königl. Regierung hat diese Bestimmung zar offentiehen Kenntniss zu bringen.

Berlin, den 31. Juli 1844.

Der Minister der Geistlichen, Unterrichts- und MedicinalAngelegenheiten.

Der Minister des Innern.

In Abwesenheit und im Auftrage:

Die eingetretene Steigerung des Preises des Jod hat eine entsprechende Erhöhung der Taxpreise dieser Drogue und der Präparate derselben nothwendig gemacht. Die königl. Regierung erhält hierb£i die demgemäss bewirkten Abänderungen der Arzneitaxe, welche mit dem 1. September d. J. in Anwendung kommen, um dieselben ungesäumt durch das Amtsblatt zur öfentlichen Kenntniss zu bringen.

Berlin, den 29. Juli 1844.

Ministerium der Geistlichen, Unterrichts- und MedicinalAngelegenheiten.

Abgeänderte Taxpreise :

Jodum................1 Scrup. $1 \mathrm{Sgr} .4 \mathrm{Pf}$.

Kali hydrojodicum .......... Drachm. $5,-$,

Tinctura Jodi............1 Drachm. 1,2 ,

Unguentum Kali hydrojodici ...1 Unze 8,6 ,

2) Ueber Mängel im Medicinalwesen; vom Apotheker Felgner.

Wenn der Hr. Müller im Archiv, Septemberheft 1843. S. 345 bei Berührung der Geschäftslosigkeit über Eingriffe der Kaufleute, Thier-

Arch. d. Pharm. XC. Bds. 1. Hft. 
ärztc, Scharfrichtcr klagt, so hätte derselbe auch die sogenannten Kỏnigseer nicht vergessen sollen - da besonders in cinem Dorfe sciner Nähe eine solche Niederlage ist, wo sclbst cin förmlicher Jlandverkauf stalb findel. - Käme dieses zu einer genauen Kenntniss einer sächsischen Medicinalbehörde, so würden nicht mehr solche Schlingpflanzen it) unserm staatsgesellschaftichen Haushalte wuchern.

Bei Entziehung des Handverkaufs in Apotheken erscheint jetz! eine Stimme des Hrn. Dr. B. in der Zeitschrift für Staatsarzneikunde, 3tes Vierteljahrheft, welche sagt, dass es niomals Arzneien gäbe, welche den Arzt unnöthig machen könnter, und doch wird Manchem einfallen, dass Patienten von wissenschaftlichen Aerzten als verloren betrachtet wurden, die später durch ein geringfügiges Nitlel hergeslellı sind - aber bei Entziehung des Handverkaufs hatte Hr. RegierungsMedicinalrath Dr. Klose wahrscheinlich nur grosse Stadte im Auge, nicht elwa, weil der Handverkauf eine Störung in der Receplur hervorbringe, denn dafür sind Recepturtisch von dem Verkaufstische getrcunt, Dr. B. meint, gewissenhafte Apothcker sollen ihren Handverkauf beschrinken - was soll nun der Apotheker kleinerer Orte angelen, sein Arzt im Orte verschreibt viclleicht täglich ein oder zwei Rerepte à 4 oder $5 \mathrm{Sgr}$. - und nicht immer hat der Arzt das Zuirauen des Publicums, und das lässt sich nicht erzwingen - denn da. wo es auf das Betragen ankommt, wollen Viele, dass sich das Publicum nach ihnen richte, sie wollen sich aber nicht nach dem Publicum genircn. (Welches angenehme Geschäft ist das Implen? - hier hal der Apothcker die Hand ganz ausser dem Spiel - man muss aber P'ublicum und Arzt hören, beide, so zu sagen, wünsehen es jedoch mil rühmlicher Ausnahme.) Sollte der Handverkauf etwa gesclimälert werden, weil das Publicum ohne Erlaubniss starkwirkende Medicamente erhalien könne, so ist hier zu bedenken, dass der Apotheker unter die grösste Controle der Aerzte selbst gestellt ist. Wenn 1)r. B. sigt, dass der Kranke oft schon wochenlang den Arzneischatz der Apotheken umsonst versucht habe, so ist zu crwahnen, dass bei schwachen Individuen der Glaube an Arcana und Wunderkuren stärker ist, als ein sicheres Heilmittel, also an reifliche, ärztliche Erfahrung und Beurthcilung, weil jene, so so sagen, etwas Individuclles, Handgreifliches haben, diese dagegen fern licgen und nur mühsam erworben werden - indem einige der Herren nicht geradezu beitragen, das Vertrauen des Publicums zu verstärken, da oft nicht bloss der College dem Collegen entgegen, nein - so zu sagen, auf den Fuss tritt. Den IIandverkauf aus der Apotheke zil verbannen, das Publicum nur an den Arzt zu weisen, um dessen Fxistenz mehr za gründen, würde in das Zeitalter des Paracelsus führen, wo in eiuem neuern Werke der Chemie angeführt wird, dass derselhe mit Kecklreit nur starkwirkende Arzncimittel anwendete; denn das Publicum hat Rechte. - Jekz sollte man wohl die Apotheken als die Controle der Aerzte betrachten? aber wir schen, nur cine Stunde von einem Städtchen entfernt, einen zum Selbstdispensiren berechligten Arzt $\longrightarrow$ der nicht etwa nur das Allernothwendigste hält, nein man trifft eine vollkommen cingerichtete $\Lambda$ potheke, welche nicht, wie dic Offein eines $\Lambda$ pothekers, der strengen Controle und Revision nuterworfen ist -.. aber auch keine Ladenhüter, wie selbiger hat. Hier wird nach Möglichkeit Alles verkauft, der äraliche Rath ist nebenbei (nur dje Medicin wird bezalilt, aber ohne die geringste Controle) und anch dispensirt - welehe Sudeleien kommen da zum Vorschein und welcho Vortheile werden da 
gchraucht! - Ich musste selbst cinmal erfahren, wie ein Landchirurg diè verordneten Recepte eines Medicus selbst verfertigte. Durch das Selbstdispensiren ist, wie schon oft erwähnt, den Pfuschern Thür und Angel geöffnet, und doch fällt es keinem Apolheker mehr ein, medicinische Praxis zu üben. Aber nicht bloss, um dem Unfug zu stevern - nein, mit Argusangen sehen die Aerzte aul die Hände des Apothekers, und bei der geringsten Uehertretung wird Anzeige gemacht und grosse Geldstrafen sind die Folgen.

Wenn sun das Publicum in die IIände eines solchen dispendirenden Arztes fältt, welcher ein Zeugniss folgender Art neulich ausstellte!

$$
\text { Z e igniss }
$$

Für der Johanne Wolfin das Selbe keine ansteckische Irützen hat, Sondern Stant es von un Reinen geblieht her.

Georgenfeld, den 12. März 1843.

G! ü ck, Arzt.

Nachdem derselbe die Patientin sehr lange behandelt hatte, behielt sie die Krätze nach wie vor. Noch kann ich orwähnen, dass cin noch jetzt lebender selbsdispensirender Chirurg rothe Corallen und Türkenfelt aus der Apotheke entnahm.

Bei Entziehung des Handverkaufs der Apotheken hatte Dr. Klose gewiss nur grosse Städte im Auge, damit sich Droguisten bilden können, die streng vom Kaufinanne getrcnnt sind -- denn weun nun die Hebamme, welche, nebenbei erwähnt, mehr auf Bildung des Volkes einwirken sollte, Ioffmannstropfen, Zimmttinctur und dergleichen gebraucht, wo soll sie sich hinwenden? - unbedingt nur an Droguisten, Dorfliämer, oder an solche, wie oben erwähnte Niederlagen der Königseer, die von Markt zu Markt ziehen und golegentlich mit ihrem medicinischen Taschenvorrath in den Dörfern hausiren - es ist doch unbedingt besser, das Publicum ist im Handverkauf an Jemand gewiesen, der, wie der Apotheker, unter der strengsten Controle steht. Wenn Dr. B. sagt, dass im Handverkauf die Waaren weit billiger als in der Receptur sind - so muss derselbe zugleich bedenken, dass der Kaufmann mit dem Apotheker fast gleiche Rechte lat, und mit ihm um die Wette laufen muss, und welchem Apotheker sollte es einfallen, z. B. gestossenen Safran oder Pulv. zingiber., wie dieselben von Hrn. K. in A. ausgeboten und bereitet werden, zur Receptur zu verwenden - nämlich zu 1 Th. Safran 2 Th., Fl. cartham, 6 Th., Lacc. viens. $\frac{3}{4}$ Th., Alumen roman. $\frac{3}{4}$ Th., Amygdal. 3 Th. Statt Pulv. zingiberi ein Gemenge aus Zingiber. 50 Th., Erbson 50 Th., Cortex cardamom. 10 Th., Piper hess. 3 Th. Nur T'üchtigkeit und Billigkeit ist dem Apotheker in seinem Fache anzurathen, denn er hat bei der Receptur eine Taxe, die er streng handhaben soll. Um Missbräuchen vorzubeugen, sollte er sich aber auch nicht weigern, die vorkommenden Mängel aufzudecken. - Die Taxen werden nicht immer, wie es sein sollte, regulirt; z. B. alś 1840 die neue Taxe erschien, war $1 \bar{z}$ Axung. porci mit 12 Pf. zu berechnen und man kaufte das Pfund mit 5 Sgr. - seit Michaelis 1842 bezahlt man das Pfd. mit 9 auch 10 Sgr., während sich der Preis in der Taxe nicht geändert hat.

Capita papaver., welche mit $2 \frac{1}{2}$ bis $3 \mathrm{Sgr}$. gekanft werden, ist die Drachme mit 3 Sgr. za berechnen.

Und wenn im Zusatz des Archivs Hr. Dr. Bley sagt, dass die Mängel durch ein geeignetes Mitglied beim Landtag in Sachsen nachgewiesen und entfernt werden würden, so kamen dicse leider diasnal nicht zur Sprache. Die Grenze zwischen liaufmam und Apothcker ist schon seit 1825 gezogen, bedarf wohl aber einer bedeutcuden Abän- 
derung? - da in denselben Droguist rom Kaufmann und Dorfkrämer geschieden sein sollte!

Der Dorfkrämer sowohl als der Kanfmann kann mit dem Apotheker Vieles gemeinschaftlich führen und in jeder Quantität verkaufen; z. B. Acet. dest. Agaricus, Axungiae. Wenn ich oben anführte, dass yon eincm noch jekt lebenden Chirurg Türkenfet versehrieben wurde, so wird es nicht auffallen, wenn es im Handverkauf noch Liebhaber finden sollte. - Grana paradis., Borax, Campher, Cubeben, Ol. de Cedro, Cassiae etc., Roob juniper. Sambue,, Manna, Minium, Lithargyr., Piper hispanii, long., einige Gummata, alle Blumen, Kräuter, Wurzeln etc. Unter $\frac{1}{4}$ Pfund ist dem Kaufmann nicht zu verkaufen gestattet, z. B. Aloc, Arcan. daplii, Bals. aopaiv., Cort. cascarill., granat., Cremor tart. und Cryst. Iart., Ot. laurin., sachar. saturn., Sal acetosell., Spir. sal. ammon., Spir. sal. tartar., auch Cassia cinnamom., und wer sollte nicht wissen, dass man wegen 1 und 3 Pf. Zimmt zum Irämer schickt - man wird aber nicht dafür 1 Pfd. bekommen! - auch wirft sich die Frage auf, wer soll darauf schen? Bis jetzt blieb immer nur dem Apothelicr die Denunciation überlassen, und soll derselbe bei seinen vielen Verantwortlichkeiten noch den Gensd'arm machen, nicht zu crwähnen, dass or sich mit seinen Mitbürgern verfeindet! - Ja man sicht das so streng verbotene Fliegenpapier bei Kaufleuten verkaufen, und in den Fenstern vieler Familien - und sind die Streichzündhölzer nicht überall gebräuchlich? - Ich bin fest überzengt, dass der Apotheker nie mit solchen verbotenen Waaren handelt; denn will er auf den Nanen cines streng rechtlichen Mannes Anspruch machen, so verschmerzt er diesen kleinen Nutzen und verfährt bei verbotenen Waren eben so streng, als bei seinem $\Lambda \mathrm{rz}$ neiwaarenhandel.

\section{Ueber Concession zum Pflasterverkauf; vom Apotheler Stresemann.}

Ueber das Keilholz'sche Pflaster ist im Junihefte des Archivs cine Mittheilung enthalten, zu der ich noch Einiges beizufügen mir erlauben will. Ein gewisser Keilholz, wie man sagt, ein ehemaliger Schauspieler, hat von der preuss. Medicinalverwaltung eine Concession erhalten zur Anfertigung eines Leichdornpflasters, mit der Bedingung, es in den Apotheken verkaufen zu lassen, wesshalb er einem hiesigen Collegen die Hauptniederlage übergeben hat. Zwar waren mit mir mehrere Collegen geneigt, beim Ministerio gegen eine solche Concession zu protestiren, indess drang unsere Stimme bei der Mehrzahl nicht durch aus dem Grunde, weil man fürchtcle, dass dieses Pflaster sodann, wie vor ihm schon mancher andere Artikel, in die Kaufläden wandern möchte, und weil man hoffte, dass es gelingen werde, nach Analogie den Kaufleuten auch die andern von ihnen geführten Medicamente zu entwinden. Ich muss gestehen, dass ich dazu wenig Hoffnung habe, bei der Unkenniniss und auch Unlust der Behörden, sich viel mit solchen Dingen zu beschäfligen.

Das in Rede stehende l'flaster wird in Kruken von etwa $1 \frac{1}{2}$ Loth Inhalt abgegeben und kostet nicht weniger als Einen Thaler.

Nach meiner Untersuchung besteht es im Wesentichen aus : Jarz, Theer, Ammoniak, Grünspan und Canthariden, letzlere in kleinen Mengen; Bleipflaster enthält es nicht. Es ist übrigens schlecht gemischt. Soweit Hr. College Stresemann. 
Wir sehen nicht ein, was das Publicum, zu dessen Nutzen doch woht die Concession gegeben sein dürte, dabei gewinnen soll; denn in jeder Apotheke ist ein ähnliches Leichdornpflaster à Unze zu 2-3 Groschen zu haben. Wenn Nichlapotheker Arzneien anferigen, so gehört das in die Reihe der Pfusehereien, zumal wenn sie so ohne alle Kunstfertigkeit gemischt sind, wie dieses in liede stehende Pflaster. Im Sinne eines guten Medicinalwesens kann es nicht liegen, aus Laien Pseudo-Apotheker zu machen. Der Pharmacie frommt nicht die Bedrückung, sondern der gecignete Schutz gegen alle Pfuschereicn von Afterärzten. Nan schützt ja doch in jeden wohlgeregelen Staale alles Eigenthum gegen fremde Eingriffe, warum soll der Apotheker allein keinen Schutz für scin Eigenthum geniessen? Er trïgl, wenn or scine Pflichten streng erfüllt, cine schwere Bürde, aber er trüge sie gern und freadig, wenn or Anerkennung, Schutz und Aufmunterung fiande. - stat dessen sicht er sich häufig Preis gegeben allen Wechsellällen von medicinischen Systemen, laxer Ausführung von schön in den Gesetzbüchern prangenden Verorilnungen and wird am Ende gar angesehen bloss als ein Zeitpächter seines mit schweren Kosten errungenen Eigenthums, das bei seinem Tode in frcmde lände übergeht. Wahrlich, ein solcher Zustand ist nicht bencidenswerth. I)as siud die Folgen des Mangels einer sachkundigen Vertretung! Ohne sie kein Ileil, ein trostloser Zustand! In ihr der Same des Gedeihens der Herstellung eines Musterinstituts in der Pharmacie!

Wir werden hingewiesen auf die Förderung der Wissenschaft, das ist schön und trefflich; denn ohne diese Basis wäre sie nichts ats ein llandwerk. Aber zur Erringung der Wissenschaft, zu ihrer Cultur sind geringe Mittel, welche kaum die Subsistenz sichern, nicht ausreichend, wer des Tages Last und Hitze getragen, bedarf auch der lirquickung, wer den wissenschaftlichen $Z$ wecken sich widmen soll, auch der geeigneten Mittel. Also vor allem eines kräftigen Schukes durch Privilegien oder vererbliche Concessionen; denn der Name thut nichts zur Sache. Die Pharmacie kann niemals der freien Concurrenz anheim. gegeben werden, wenn man nicht Heil in Unheil verkehren will. Dic Geschichte der Pharmacie kann dalür Beläge geben, wohin die freie Concurrenz führt, zur Pfuscherei, zur Untergrabung alles wissenschaftlichen Strcbens. Ferner strenge Prüfungen nach streng geregeltcr wissenschaftlicher Ausbildung, kräftiges Handhaben guter medicinalpolizeilicher (iesetze nebst Abstellung aller Pfuschereicn, also ungestörtes Ausüben des pharmacentischen Berufes in der Ausdehnung auf alle Arzneimittel, sic mögen auf allöopathische oder homöopathische Verordnungen sich stülzen, für Menschen oder Thiere bestimmt seien. Dieses ist es, was die Pharmacie will, was ihr Noth thut, was sie nach Gerechtigkeit und Billigkeit verlangen kann und verlangen muss, dann aber werden auch ohne weitere Beihülfe des Staats Talent und Thätigkeit allenthalben sich Buhn brechen, die eine eben so grosse und wohl noch ehrenwerthere Macht bilden, als die Geldmachl es ist! Dic Folge aber wird sich zeigen in dem erneuten Aufwachen wissenschaftlichen Sinnes in der Erzeugung schöner Früchte gediegenen Wissens. Möge die Erleuchtung da nicht fehlen, wo sie Noth that, um zu bescheinen das Bild der künstlich erzeugten Verdunkelung, und möge sie bald eincm hellen Tage Platz machen!

Dr. Bley. 